\title{
Imaging patterns of liver uptakes on PET scan: pearls and pitfalls
}

Wing Hang Luk, Andrea Wai San Au-Yeung,

Tony Kwok Loon Loke

Department of Radiology, United Christian Hospital, Hong Kong SAR

[Received 29 I 2013; Accepted 10 VI 2013]

\section{Abstract}

Objective: PET imaging is becoming increasingly universal, and therefore increased liver uptake is frequently encountered. The purpose of this article is to describe and illustrate the various morphological patterns of increased metabolic activity within the liver with an emphasis on the diagnostic pitfalls and potential limitations.

Conclusion: Knowing the pitfalls of PET imaging, correlation with clinical background and findings from other imaging modalities are all important in the correct interpretation of increased hepatic activity on PET imaging.

KEY words: liver, PET, uptakes pattern

Nuclear Med Rev 2013; 16, 1: 75-81

\section{Introduction}

Positron emission tomography (PET) has emerged as an increasingly important functional diagnostic tool in a wide spectrum of conditions in recent years. By far the most extensive use of $\mathrm{PET} / \mathrm{CT}$ imaging to date has been in the diagnosis, staging and monitoring of malignancies. As liver is the one of the commonest sites of metastasis for tumours arising from the gastrointestinal tract, lung, breast, pancreas and melanoma etc. [1, 2] and that the presence of hepatic metastasis often significantly alters treatment plan, liver imaging presents a common challenge in oncological evaluation by PET. In the contrary, other imaging modalities such as ultrasound (US), dynamic CT or contrast-enhancement MRI remain the mainstays for diagnosis of primary liver tumours such as hepatocellular carcinomas (HCC). Nevertheless, occasion

Correspondence to: Wing Hang Luk

Department of Radiology, United Christian Hospital

B1/F, Block S, 130 Hip Wo Street, Kwun Tong, Kowloon, Hong Kong SAR

Tel.: (0852) 35134156

Fax: (0852) 35135637

E-mail: lukwinghang@gmail.com arises where whole-body PET/CT is employed as supplementary tool in the characterisation of known primary liver lesions or for various inflammatory/infective conditions. Recognizing the different morphological patterns of increased liver uptake is therefore imperative.

\section{PET tracers}

18-fluoro-2-deoxyglucose (FDG), an analogue of glucose that allows evaluation of glucose metabolism, is the most commonly used PET tracer. FDG enters cells by glucose transporters and is intracellularly phosphorylated by hexokinase into FDG-6-phosphate. However unlike glucose, it cannot be further metabolised and therefore becomes "trapped" within the cell in a manner proportional to the glycolytic rate. The presence of facilitated glucose transporters, especially type 1 (GLUT1) and hexokinase II, in many forms of solid malignancies and the consequent accelerated glycolytic rate is exploited in FDG-PET imaging.

In some types of malignancy however, this alteration in tumour kinetics is not seen. HCCs for instance, may exhibit a higher glucose-6-phosphatase activity to phosphorylation kinase activity ratio, thereby resulting in a low glucose metabolism and FDG uptake - a fact that is well recognized for the well-differentiated and lower grade tumours [3]. In this regard, Carbon-11 acetate serves as an important complementary metabolic agent and will be discussed in more detail later.

In this review article, we aimed to describe and illustrate the different morphological patterns of hepatic uptake that may be seen with these two PET tracers.

\section{Potential diagnostic pitfalls}

Before discussing the various patterns of increased hepatic uptake, it is useful to first consider the potential diagnostic pitfalls in PET imaging.

Apart from the general technology-specific pitfalls like misregistration, attenuation correction and truncation artefacts etc. [4], there are imaging issues that specifically relate to the liver. Normal liver, like the spleen, is a site of diffuse mild to moderate FDG uptake [5]. The usual appearance of liver parenchyma is that of a mildly intense, uniformly mottled appearance. This makes any increased uptake related to a focal lesion more difficult to be discerned from the background physiological activity. In an attempt to increase conspicuity and detection rate of liver metastasis, dual-time-point 


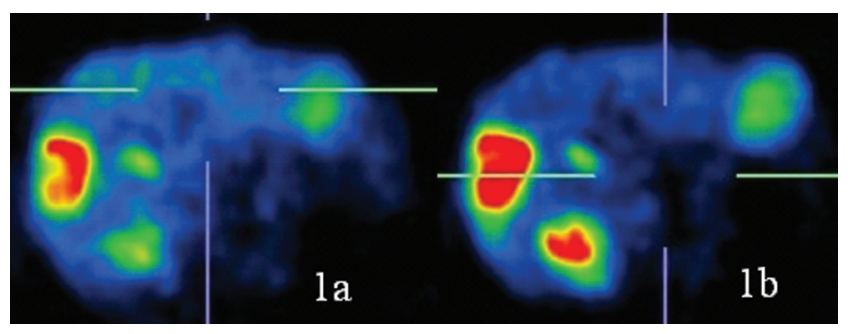

Figure 1. 56-year-old man with carcinoma of colon and liver metastasis. A. Axial image of the FDG PET at 90 minutes scan has higher background liver activity compared with the 120 minutes delayed scan image B. (SUVmax 9.6, arrow; SUVmax 11.7, arrowhead)

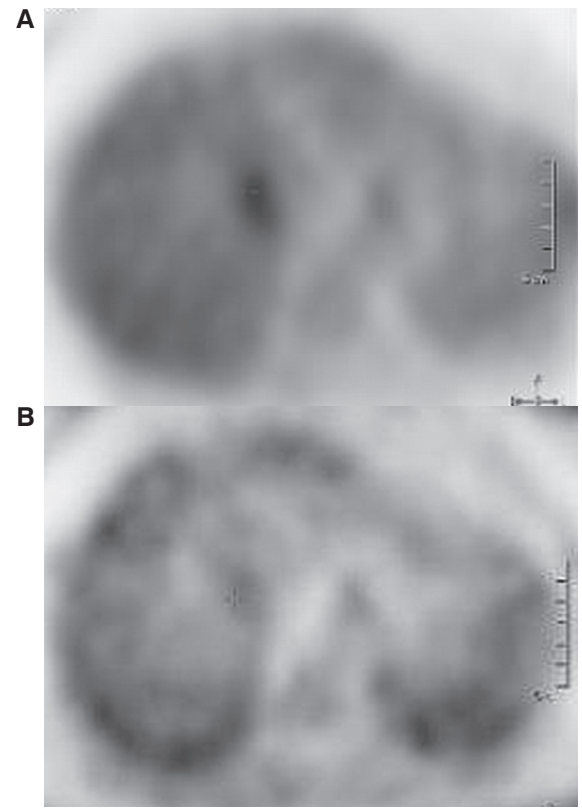

Figure 2. 58-year-old woman with carcinoma of rectum. A. Longer scanning time (4 minutes per bed position) shows segment 8 HCC not seen on normal scanning time with mild increased metabolic activity (SUVmax 2.5) as compared with adjacent liver parenchyma (SUVmax 2.2); B. Normal scanning time (2 minutes per bed position)

FDG-PET imaging with delayed acquisition at 120mins has been used by some centres with success. It has been shown to be more sensitive in detecting liver metastases, revealing new lesions in 17\% of patients when compared with standard imaging alone [6]. The improved performance is believed to be due to altered tumour vascularity and better clearance of blood-pool activity with time (Fig. 1) [7] Another technique that has been proposed to improve image quality is the lengthening of image acquisition time (Fig. 2) [8].

Size is another factor that will affect the sensitivity of PET imaging. Studies have indicated that PET is most accurate for detection of hepatic metastases greater than $1 \mathrm{~cm}$ in diameter, but becomes limited when lesions are smaller than $1 \mathrm{~cm}[9,10]$.

Age may also have a significant and positive impact on both maximum and mean standardized uptake values of the liver, as suggested by Lin et al. [11].

Furthermore, hepatic steatosis may potentially alter hepatic metabolic activity on PET imaging although the difference is considered too small to be significant [12].
Table 1. Differential diagnosis of liver uptake lesions in FDG PET

$\begin{array}{ll}\text { Infection and inflammatory } & \text { Abscess } \\ \text { lesion } & \\ \text { Sarcoidosis } & \\ \text { Histiocytosis } & \\ \text { Pseudotumour } & \text { Adenoma } \\ \text { Benign tumour } & \text { Hepatocellular carcinoma } \\ \text { Fibronodular hyperplasia } & \\ \text { Malignant tumour } & \\ \text { Cholangiocarcinoma } & \\ \text { Metastasis } & \\ \text { Lymphoma } & \\ \text { Hepatic angiosarcoma } & \end{array}$

\section{Morphological patterns on FDG-PET imaging}

\section{Solitary uptake}

Solitary uptake in the liver is non-specific in PET imaging (Table 1) [13]. It can be due to infection (Fig. 3) or other inflammatory conditions like sarcoidosis [14], histiocytosis [15] and pseudotumour [16]. FDG is well known to accumulate in inflammatory cells such as lymphocytes, neutrophils and macrophages in various inflammatory or infectious conditions due to elevated glucose requirements [17]. Hence one should be aware of potential false positive results in such conditions

Primary liver tumours, either benign, malignant or metastastic (Fig. 4) are frequently encountered in oncological imaging. This emphasises the need for other imaging modalities in the evaluation of liver masses [18]. Benign liver tumours like haemangioma, focal nodular hyperplasia $(\mathrm{FNH})$, and $\mathrm{HCC}$ have been shown to generally take up FDG at a similar rate as normal liver tissue [19]. Nevertheless, there have been case reports of increased uptake by $\mathrm{FNH}$ [20] and hepatocellular adenoma [21]. For malignant primary liver tumours in particular HCC, the sensitivity of FDG-PET is less than remarkable - a reported range of 50-65\% [22, 23]. As for cholangiocarcinoma, the sensitivity of FDG PET is high (>90\%) for the intrahepatic subtype and less so for the extrahepatic subtype (about 60\%) [24]. Uncommon liver malignancies like lymphoma, both primary [25] and secondary [26], and angiosarcoma [27] have also been reported to show increased FDG uptakes.

With regards to liver metastases, several types are known to have avid FDG uptake and thus FDG-PET generally has a good detection rate [28]. Kinkel et al. [29] found that FDG-PET is the most sensitive (90\%) noninvasive imaging modality for the diagnosis of hepatic metastases from colorectal, gastric and oesophageal cancers. A recent meta-analysis demonstrated the sensitivities of $C T$, MR and FDG-PET for detecting hepatic metastases from colorectal carcinoma to be $83.6 \%, 88.2 \%$, and $94.1 \%$, respectively [30]. But if lesions are smaller than $1 \mathrm{~cm}$ in diameter, the sensitivity may be lower as previously explained [31, 32].

\section{Ring-like uptake}

Ring-like FDG uptake is also non-specific but in the context of oncological imaging, it may indicate mucin-producing tumours like cholangiocarcinoma (Fig. 5) or metastasis from adenocarcinoma [33] Mucin-producing neoplasm such as mucinous colorectal carci- 


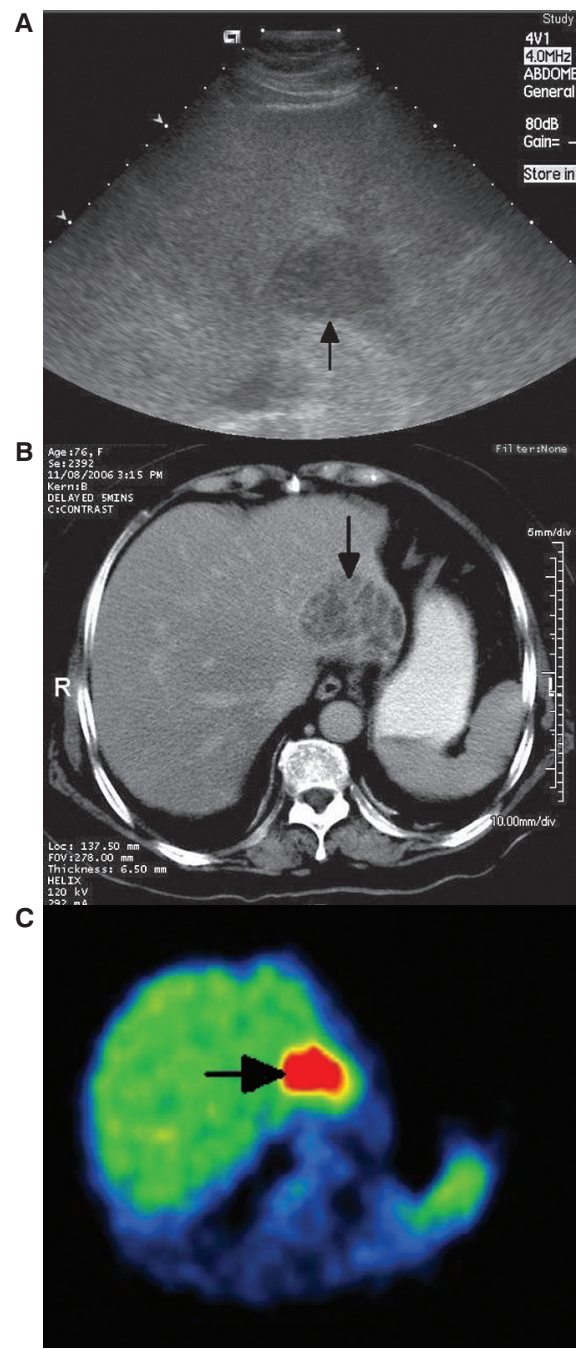

Figure 3. 76-year-old woman presented with fever of unknown origin. A. Ultrasound shows a hypoechogenic lesion in the left lobe of the liver (arrow); B. Axial post contrast CT demonstrates a heterogeneous enhancing lesion (arrow); C. Patient refused biopsy and PET/CT was offered. Axial image of the FDG PET shows the corresponding lesion with increased metabolic activity SUVmax 9.3 (arrow). The patient was treated with antibiotics and follow-up CT showed complete resolution of the lesion confirming an abscess

noma contains abundant mucin and often exhibit significantly lower peak standardized uptake values. This is believed to be secondary to a lower glucose metabolic rate and cellularity [34]. Presence of necrosis is another reason for ring-like uptake (Fig. 6). Intra-acinar necrosis may be seen in colorectal carcinoma [35] and can give rise to significant decrease in FDG uptake. Last but not least, ring-like FDG uptake within the liver is also seen in inflammatory conditions such as large liver abscess with liquefaction centre [13], or acute cholecystitis [36].

\section{Multiple uptakes}

In oncological imaging, multiple sites of focally increased FDG concentration in the background of relatively lower normal hepatocyte uptake are generally regarded as the hallmark of metastatic liver involvement from a known primary tumour (Figs. 7 and 8).

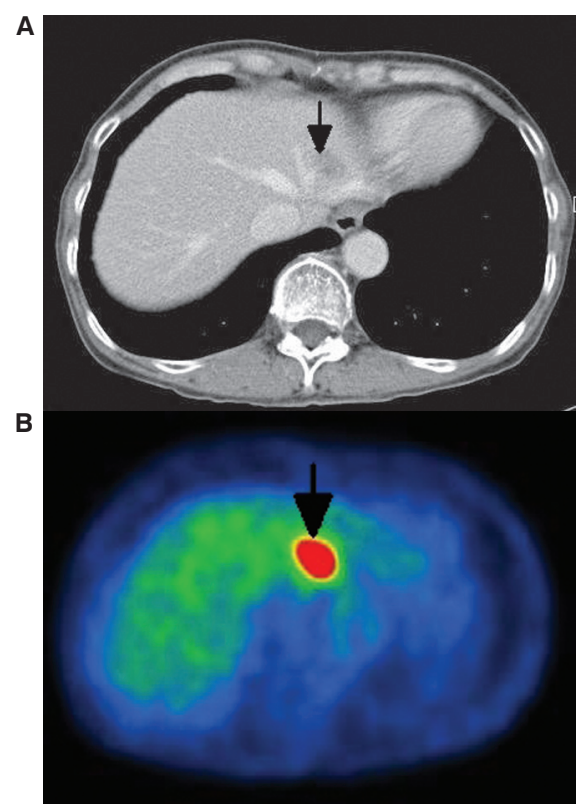

Figure 4. 69-year-old woman with carcinoma of rectum. A. Axial contrast CT shows a hypoenhancing lesion in segment 2 of the liver (arrow) with corresponding (B) FGD PET showing a lesion with increased metabolic activity lesion (SUVmax 13) (arrow). Histopathology confirmed liver metastasis from carcinoma of rectum after segmentectomy

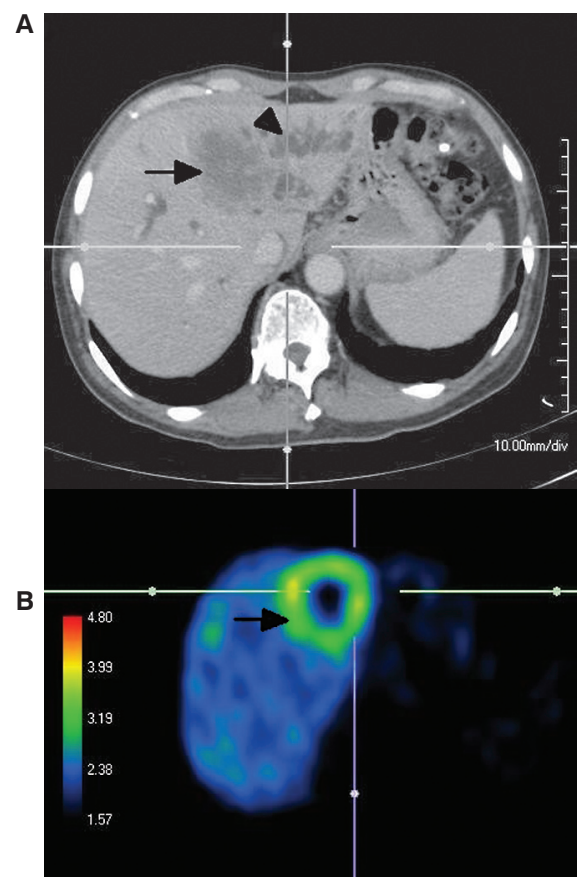

Figure 5. 67-year-old man with cholangiocarcinoma. A. Axial contrast CT shows a tumour in the left lobe of liver (arrow) with ductal dilation (arrow head); B. The left lobe lesion had a large photopenic centre with a rim of marked uptake (SUVmax 5.2) (arrow) on axial image FDG PET. Histology confirmed moderately to poorly differentiated cholangiocarcinoma after left hepatectomy 


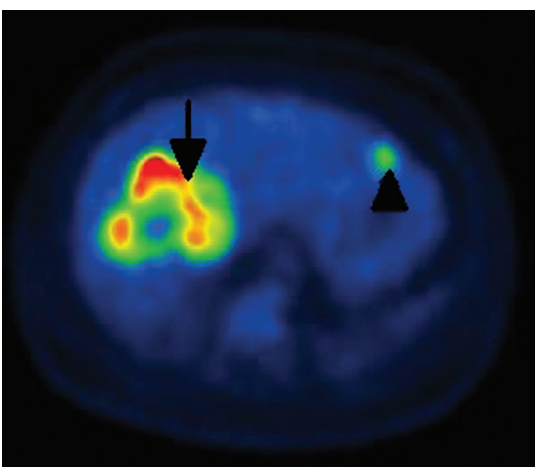

Figure 6. 72-year-old man with carcinoma of colon. The lesion is in right lobe of the liver (arrow) with increased metabolic activity predominantly in the periphery (SUVmax 12.8) on axial FDG PET. A smaller increased metabolic activity focus (arrow head) is seen in the lateral segment of the liver (SUVmax 5.3). Biopsy confirmed liver metastasis from carcinoma of colon with central necrosis
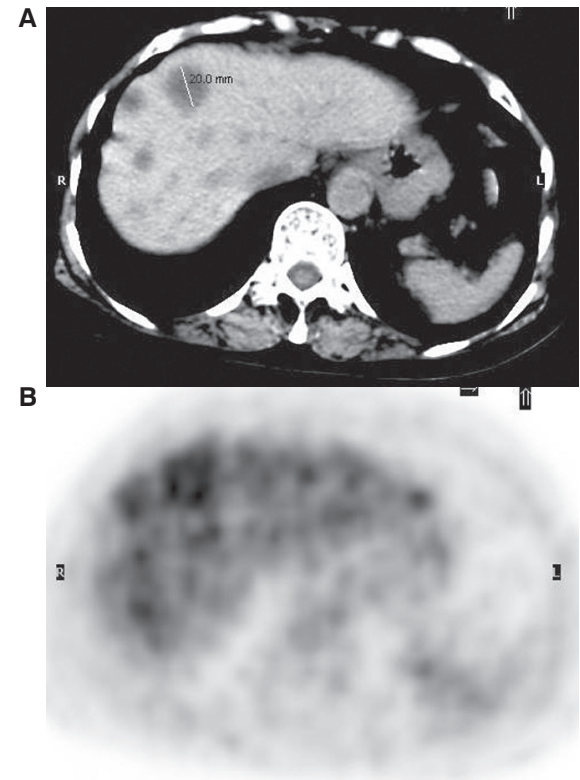

Figure 7. 79-year-old woman with carcinoma of colon. A. Axial non-contrast CT shows multiple hepatic hypoattenuated lesions with sized up to $2 \mathrm{~cm}$; $\mathbf{B}$. Axial FDG PET of the liver shows multiple increased metabolic activity with highest SUVmax 5.3 for the corresponding biggest lesion on CT. Features are compatible with multiple liver metastases. The patient succumbed one month after the PET/CT due to liver failure

However, the differential diagnosis of liver uptake (Table 1) is still applicable and that correlation with clinical setting and other imaging findings is essential for the correct diagnosis.

\section{Diffusely increased liver uptake ("Hepatic superscan")}

"Hepatic superscan" is an uncommon but interesting finding in FDG-PET imaging. Analogous to the superscan seen in conventional skeletal scintrigraphy, it describes the appearance of intense diffuse hepatic tracer uptake, often coupled with surprisingly low brain and cardiac FDG uptake. This appearance can be seen in diffuse extensive involvement by malignancy like hepatic lymphoma [37], HCC or metastasis [38]. Similar diffuse increase in liver FDG

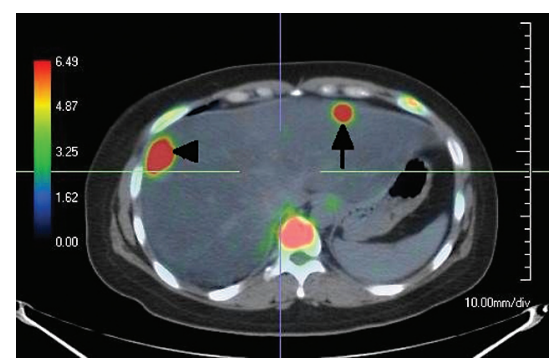

Figure 8. 33-year-old woman with carcinoma of breast. Fused axial FDG PET/CT shows marked focal increased metabolic activity in the segment 3 (arrow) and 5 (arrow head), with SUVmax 21.0 and 15.0 respectively. Follow-up contrast CT showed enlargement of the two lesions confirming the diagnosis of liver metastasis

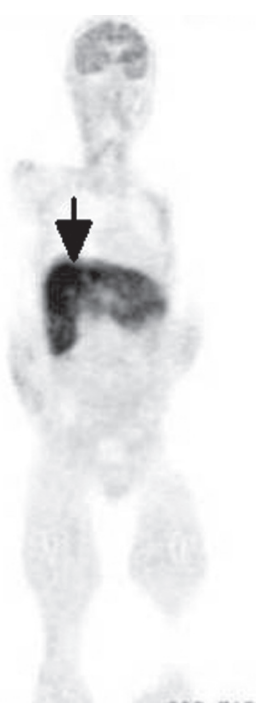

Figure 9. 46-year-old-man with history of fever of unknown origin. Axial FDG PET/CT shows diffuse intense homogenous hypermetabolic activity in the liver (SUVmax 7.1) (arrow). Liver biopsy showed acid fast bacilli confirming the diagnosis of primary liver tuberculosis

uptake from diffuse involvement by tuberculosis has been described as "hot liver" (Fig. 9) [39].

\section{Carbon-11 acetate PET}

As discussed above, the role of FDG-PET in the detection and diagnosis of HCC is limited. Elevated FDG uptake in malignant tumours largely depends on the presence of facilitated glucose transporters and glycolytic enzyme hexokinase II but this alteration in kinetic pathways does not occur in all forms of solid malignancy. $\mathrm{HCC}$ is one of such examples. As mentioned previously, many HCCs particularly those that are well-differentiated and lower grade exhibit a lower FDG avidity and may escape detection on FDG-PET imaging (Fig. 10). It is therefore not surprising that the general reported false-negative rate using FDG-PET in the imaging of primary HCC only approaches $50-65 \%$ [40, 41].

The use of Carbon-11 acetate as dual tracer is now well established in the detection of HCC. The exact uptake mechanism of Carbon 11 is not known, but studies suggest that it is incorporated into the lipid pool in cancer tissue with low oxidative metabolism and high lipid synthesis [42]. An early study demonstrated that 


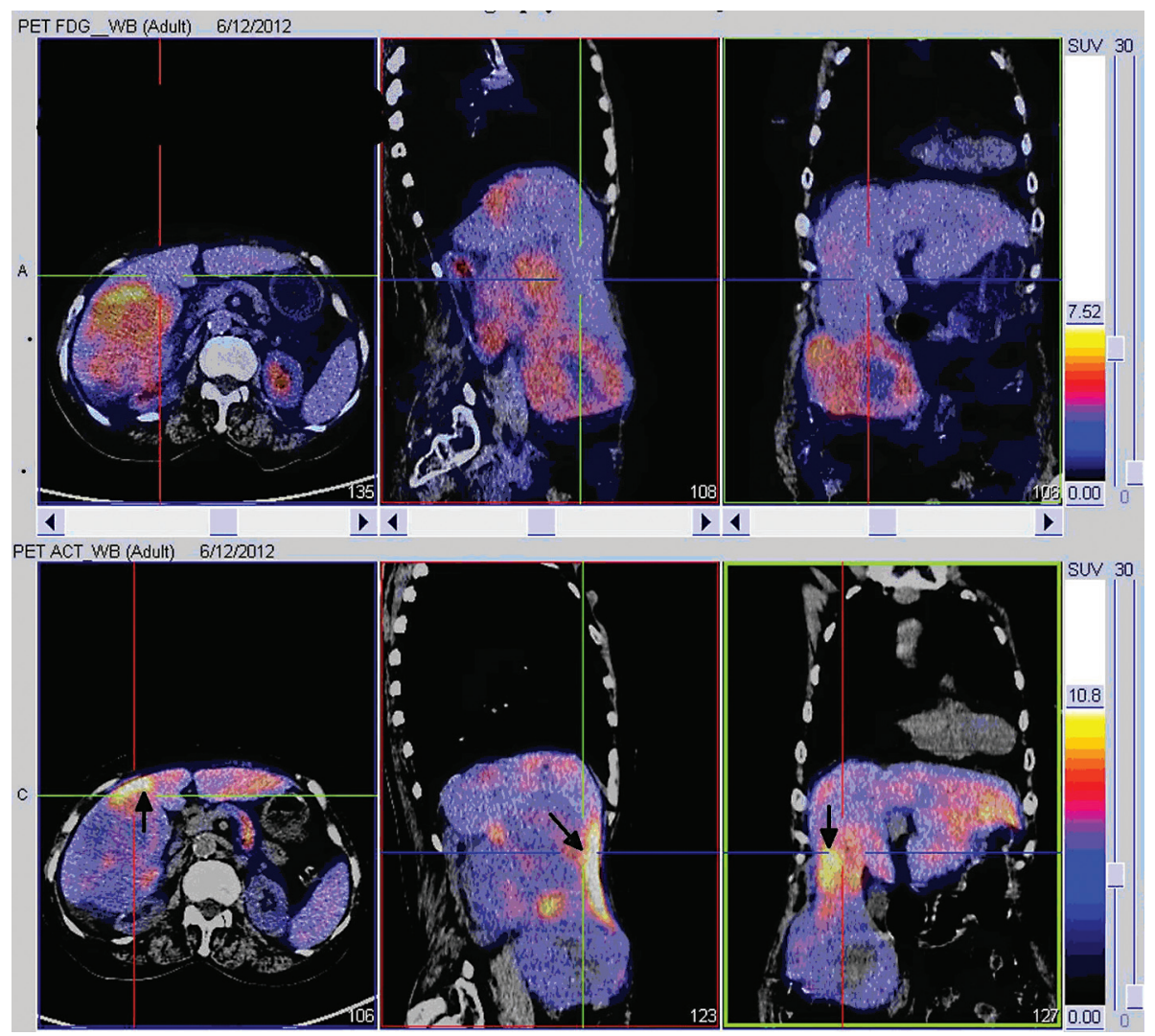

Figure 10. 76-year-old woman with markedly elevated alpha fetoprotein (> $60500.0 \mathrm{ug} / \mathrm{L}$ ). Dual-tracer multi-planar images from fused FDG PET/CT (upper row) shows negative FDG uptake in the segment V/VIII but positive 11C-acetate PET/CT (lower row) uptake (SUVmax 13.2) (arrow heads). Histology confirmed HCC

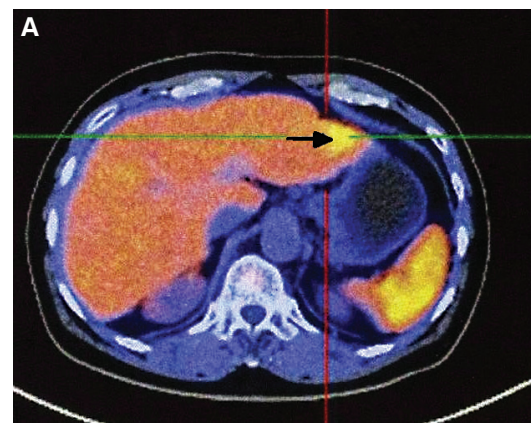

B

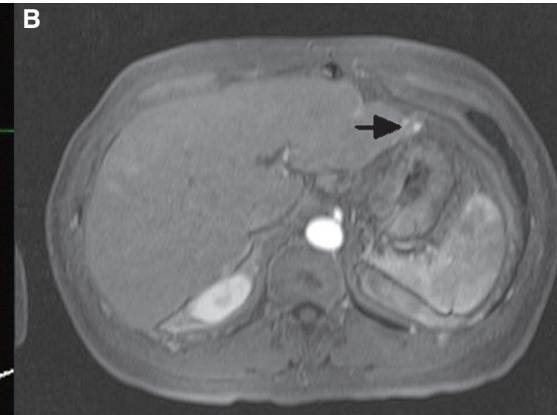

C

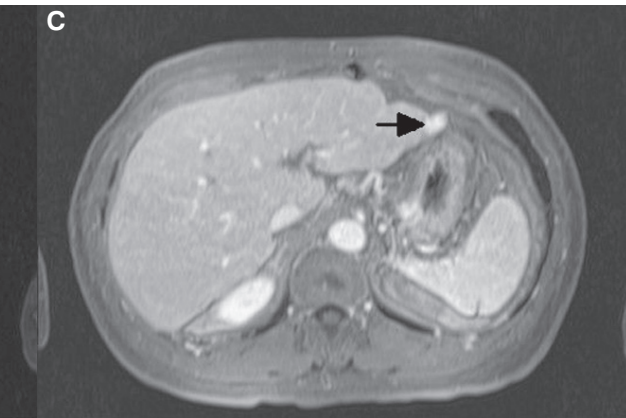

Figure 11. A 76-year-old man with resected segment 6 HCC. A. Follow-up dual-tracer multi-planar images from fused 11C-acetate PET/CT shows an increased metabolic activity area (SUVmax 8.7) at segment 3 of the liver (arrow); B. Axial post-contrast arterial phase CT shows a small hypervascular lesion (arrow); C. Axial post-contrast shows delayed filling of contrast on portal venous phase (arrow) consistent with a haemangioma. Follow-up CT at six months showed similar findings with no enlargement of the lesion

Carbon-11 acetate has sensitivity for detection of HCC of $87.3 \%$ while that of $18 \mathrm{~F}-\mathrm{FDG}$ was $47.3 \%$. When both tracers were used, they appeared to be complementary, giving a specificity of $100 \%$ [43]. As a single agent, Carbon-11 acetate appears to be more sensitive in detecting primary HCC that $18-F$ FDG $(75.4 \%$ vs. $60.9 \%)$ although the converse is true in the case of metastatic HCC $(77 \%$ vs. 85.7\%) [44]. Carbon-11 acetate is therefore considered by many as complementary to $18-\mathrm{F} \mathrm{FDG} \mathrm{in} \mathrm{HCC} \mathrm{imaging.}$

Nonetheless, it has been shown that false positive Carbon-11 acetate uptake may occur in a variety of non-HCC hepatic le- sions e.g. angiomyolipoma [45] and haemangioma (Fig. 11). In analogy with the use of Carbon-11 acetate in the evaluation of -oxidative metabolism of the myocardium [46], the large smooth muscular component within this unusual hepatic lesion might have accounted for the high uptake of Carbon-11 acetate in haemangioma. One study showed that Carbon-11 acetate is not useful in distinguishing $\mathrm{HCC}$ from $\mathrm{FNH}$ [47]. In our experience, increased Carbon-11 acetate uptake due to presence of arterioportal shunts which are artefacts are not uncommon in cirrhotic livers and can be easily mistaken for HCC (Fig. 12). 


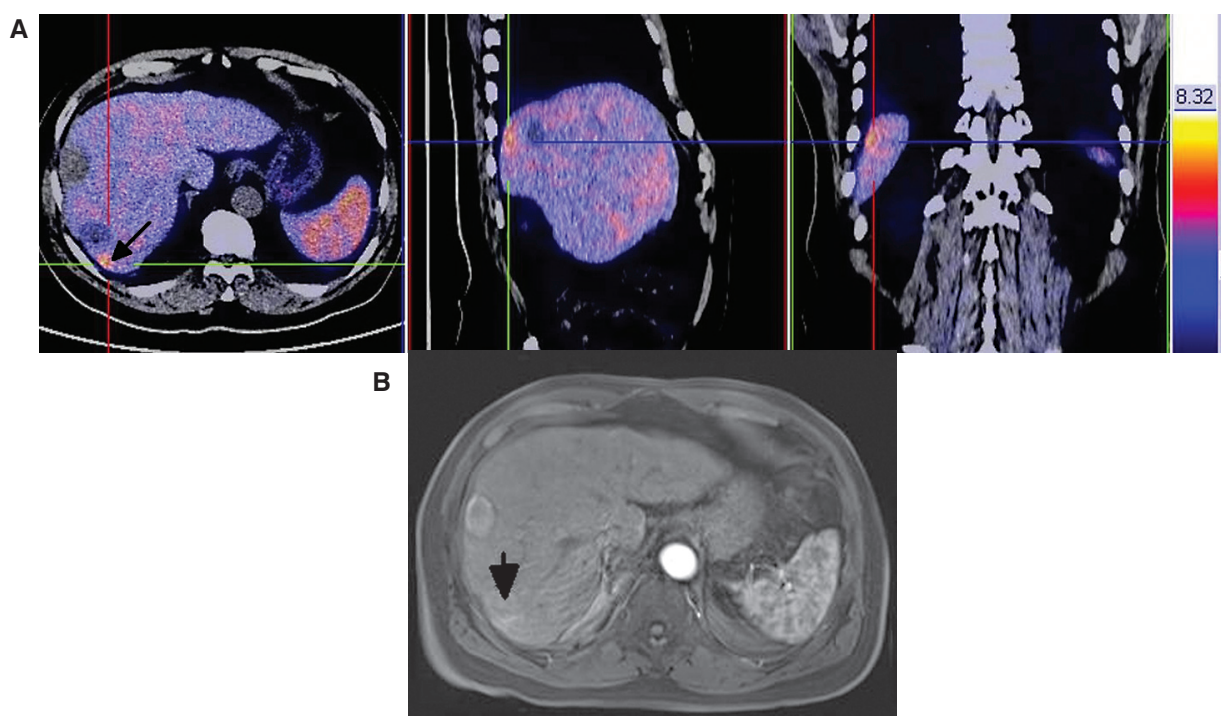

Figure 12. 66-year-old man with history of HCCs and RFA treatment at segment 7 and 8. A. Dual-tracer multi-planar images from fused 11C-acetate PET/CT shows a small focal increased metabolic activity lesion (SUVmax 7.7) (arrow) at segment 7 of the liver, posterior to the previous RFA site; B. Axial post-contrast MRI image of the liver shows a wedge shape enhancing area (arrow) corresponding to the Carbon 11 acetate avid area (due to the present of arteroportal venous shunt). Follow-up MRI twelve months later showed no evidence of HCC

\section{Conclusion}

Abnormal liver uptake in PET imaging is a common finding. Different patterns of liver uptake have been described and none of them are specific to any underlying pathology. Knowing the pitfalls of PET imaging, correlation with clinical background and findings from other imaging modalities is the key to correctly interpreting altered hepatic activity. In doubtful cases, histological confirmation may still be required.

\section{References}

1. Bengmark $S$, Hafstrom $L$, Olssen A et al. The natural history of primary and secondary liver tumours. $V$. The prognosis for conventionally treated patients with liver metastases. Digestion 1972; 6: 321-329.

2. American Cancer Society. Cancer Facts and Figures, 1999. Atlanta, Ga: American Cancer Society; 1999.

3. Okazumi $\mathrm{S}$, Isono $\mathrm{K}$, Enomoto $\mathrm{K}$ et al. Evaluation of liver tumors using fluorine-18-fluorodeoxyglucose PET: characterization of tumor and assessment of effect of treatment. J Nucl Med 1992; 33: 333-339.

4. McDermott S, Skehan SJ. Whole body imaging in the abdominal cancer patient: pitfalls of PET-CT Abdom Imaging 2010; 35: 55-69. Epub 2009 Jan 7.

5. Shreve PD, Anzai Y, Wahl RL. Pitfalls in oncologic diagnosis with FDG PET imaging: physiologic and benign variants. RadioGraphics 1999; 19: 61-77.

6. Kuker RA, Mesolaras G, Gulec SA. Optimization of FDG-PET/CT imaging protocol for evaluation of patients with primary and metastatic liver disease. Int Semin Surg Oncol 2007; 4: 17.

7. Dirisamer A, Halpern BS, Schima W et al. Dual-time-point FDG-PET/CT for the detection of hepatic metastasis. Mol Imaging Biol 2008; 10: 335-340.

8. Masuda $\mathrm{Y}$, Kondo C, Matsuo $\mathrm{Y}$, Uetani M, Kusakabe K. Comparison of imaging protocols for 18F-FDG PET/CT in overweight patients: optimizing scan duration versus administered dose. J Nucl Med 2009; 50: 844-848. Epub 2009 May 14

9. Fong $Y$, Saldinger PF, Akhurst T et al. Utility of $18 \mathrm{~F}-\mathrm{FDG}$ positron emission tomography scanning in selection of patients for resection of hepatic colorectal metastases. Am J Surg 1999; 178: 282-287.
10. Delbeke D, Vitola JV, Sandler MP et al. Staging recurrent metastatic colorectal carcinoma with PET. J Nucl Med 1997; 38: 1196-1201.

11. Lin CY, Ding HJ, Lin CC, Chen CC, Sun SS, Kao CH. Impact of age on FDG uptake in the liver on PET scan. Clin Imaging 2010; 34: 348-350.

12. Abikhzer G, Alabed YZ, Azoulay L, Assayag J, Rush C. Altered hepatic metabolic activity in patients with hepatic steatosis on FDG PET/CT. AJR Am J Roentgenol 2011; 196: 176-180.

13. Delbeke D, Martin WH, Sandler MP, Chapman WC, Wright JK Jr, Pinson CW. Evaluation of benign vs malignant hepatic lesions with positron emission tomography. Arch Surg 1998; 133: 510-515; discussion 515-6.

14. Guglielmi AN, Kim BY, Bybel B, Slifkin N. False-positive uptake of FDG in hepatic sarcoidosis. Clin Nucl Med 2006; 31: 175.

15. Hu X, Dong A, Lv S. F-18 FDG PET/CT imaging of solitary liver Langerhans cell histiocytosis: preliminary findings. Ann Nucl Med 2012; Mar 8. Epub ahead of print.

16. Kawamura E, Habu D, Tsushima $\mathrm{H}$ et al. A case of hepatic inflammatory pseudotumor identified by FDG-PET. Annals of Nuclear Medicine 2006; 20: 321-323.

17. Weisdorf DJ, Craddock PR, Jacob HS. Glycogenolysis versus glucose transport in human granulocytes: differential activation in phagocytosis and chemotaxis. Blood 1982; 60: 888-893

18. McDermott $\mathrm{S}$, Skehan SJ. Whole body imaging in the abdominal cancer patient: pitfalls of PET-CT Abdom Imaging 2010; 35: 55-69. Epub 2009 Jan 7.

19. Sacks A, Peller PJ, Surasi DS, Chatburn L, Mercier G, Subramaniam RM. Value of PET/CT in the Management of Primary Hepatobiliary Tumors, Part 2. AJR Am J Roentgenol 2011; 197: W260-265.

20. Shiomi S, Kurooka H, Iwata Y. Two cases of focal nodular hyperplasia of the liver: value of scintigraphy with Tc-99m GSA and positron emission tomography with FDG. Ann Nucl Med 1999; 13: 427-431.

21. Stephenson JA, Kapasi T, Al-Taan O, Dennison AR. Uptake of 18FDG by a Hepatic Adenoma on Positron Emission Tomography. Case Reports in Hepatology Vol 2011, Article ID 276402.

22. Roh MS, Jeong JS, Kim YH, Kim MC, Hong SH. Diagnostic utility of GLUT1 in the differential diagnosis of liver carcinomas. Hepatogastroenterology 2004; 51: 1315-1318.

23. Khan MA, Combs CS, Brunt EM et al. Positron emission tomography scanning in the evaluation of hepatocellular carcinoma. J Hepatol 2000; 32: 792-797. 
24. Breitenstein S, Apestegui C, Clavien PA. Positron emission tomography (PET) for cholangiocarcinoma. HPB (Oxford) 2008; 10: 120-121.

25. Seshadri N, Ananthasivan R, Kavindran R, Srikanth G, Chandra S. Primary hepatic (extranodal) lymphoma: utility of [(18)F]fluorodeoxyglucose-PET/CT. Cancer Imaging 2010; 10: 194-197.

26. Lin E, Lee M, Agoff N. FDG PET/CT diagnosis of hepatic lymphoma mimicking focal fatty infiltration on CT. J Radiol Case Rep 2010; 4: 34-37. Epub 2010 Apr 1.

27. Maeda T, Tateishi U, Hasegawa T, Ojima H, Arai Y, Sugimura K. Primary Hepatic Angiosarcoma on Coregistered FDG PET and CT Images. AJR Am J Roentgenol 2007; 188: 1615-1617.

28. Sacks A, Peller PJ, Surasi DS, Chatburn L, Mercier G, Subramaniam RM. Value of PET/CT in the Management of Liver Metastases, Part 1. AJR Am J Roentgenol 2011; 197: W256-259.

29. Kinkel K, Lu Y, Both M, Warren RS, Thoeni RF. Detection of hepatic metastases from cancers of the gastrointestinal tract by using noninvasive imaging methods (US, CT, MR imaging, PET): a meta-analysis. Radiology 2002; 224: 748-756.

30. Niekel MC, Bipat S, Stoker J. Diagnostic imaging of colorectal liver metastases with CT, MR imaging, FDG PET, and/or FDG PET/CT: A meta-analysis of prospective studies including patients who have not previously undergone treatment. Radiology. 2010; 257: 674-684. Epub 2010 Sep 9.

31. Rohren EM, Paulson EK, Hagge R et al. The role of F-18 FDG positron emission tomography in preoperative assessment of the liver in patients being considered for curative resection of hepatic metastases from colorectal cancer. Clin Nucl Med 2002; 27: 550-555.

32. Sahani DV, Kalva SP, Fischman AJ, et al. Detection of liver metastases from adenocarcinoma of the colon and pancreas: comparison of mangafodipir trisodium-enhanced liver MRI and whole-body FDG PET. AJR Am J Roentgenol 2005; 185: 239-246.

33. Au-Yeung AW, Luk WH, Lo AX. Imaging features of colorectal liver metastasis in FDG PET-CT: a retrospective correlative analysis between CT attenuation and FDG uptake. Nucl Med Commun 2012; 33: 403-407.

34. Berger KL, Nicholson SA, Dehdashti F, Siegel BA. FDG PET evaluation of mucinous neoplasms: correlation of FDG uptake with histopathologic features. AJR Am J Roentgenol 2000; 174: 1005-2000
35. Redston M. Epithelial neoplasms of the large intestine. In: Odze RD, Goldblum JR, editors.Surgical pathology of the GI tract, liver, biliary tract and pancreas. 2nd ed. Philadelphia, PA: Saunders/Elsevier 2009: 597-637.

36. Kao CH. Ring-like FDG uptake in acute cholecystitis. Clin Nucl Med 2003: 28: 162-163.

37. Kaneko K, Nishie A, Arima F. A case of diffuse-type primary hepatic lymphoma mimicking diffuse hepatocellular carcinoma. Ann Nucl Med 2011; 25: 303-307. Epub 2011 Jan 14.

38. Tichelaar V, Gemmel F, de Rhoter W, Bronkhorst C, de Graaf H. FDG hepatic superscan caused by massive breast cancer invasion. Clin Nucl Med 2009; 34: 716-718.

39. Jeong YJ, Sohn MH, Lim ST et al. 'Hot liver' on 18F-FDG PET/CT imaging in a patient with hepatosplenic tuberculosis. Eur J Nucl Med Mol Imaging 2010; 37: 1618-1619. Epub 2010 May 27.

40. Roh MS, Jeong JS, Kim YH, Kim MC, Hong SH. Diagnostic utility of GLUT1 in the differential diagnosis of liver carcinomas. Hepatogastroenterology 2004; 51: 1315-1318.

41. Khan MA, Combs CS, Brunt EM et al. Positron emission tomography scanning in the evaluation of hepatocellular carcinoma. J Hepatol 2000; 32: 792-797.

42. Park JW, Kim JH, Kim SK et al. A prospective evaluation of 18 F-FDG and 11C-acetate PET/CT for detection of primary and metastatic hepatocellular carcinoma. J Nucl Med 2008; 49: 1912-1921.

43. Ho CL, Yu SC, Yeung DW. 11C-acetate PET imaging in hepatocellular carcinoma and other liver masses. J Nucl Med 2003; 44: 213-221.

44. Park JW, Kim JH, Kim SK et al. A prospective evaluation of 18F-FDG and 11C-acetate PET/CT for detection of primary and metastatic hepatocellular carcinoma. J Nucl Med 2008; 49: 1912-1921. Epub 2008 Nov 7.

45. Lhommel R, Annet L, Bol A. PET scan with 11C-acetate for the imaging of liver masses: report of a false positive case. Eur J Nucl Med Mol Imaging 2005; $32: 629$

46. Henes CG, Bergmann SR, Walsh MN, Sobel BE, Geltman EM. Assess-ment of myocardial oxidative metabolic reserve with positron emission tomography and carbon-11 acetate. J Nucl Med 1989; 30: 1489-1499.

47. Grassi I, Nanni C, Allegri V et al. The clinical use of PET with 11C-acetate. Am J Nucl Med Mol Imaging 2012; 2: 33-47. 